\title{
Pro-inflammatory cytokine release by peripheral blood mononuclear cells from patients with advanced pancreatic cancer: Relationship to acute phase response and survival
}

\author{
ALASTAIR G.W. MOSES, JEAN MAINGAY, KATHRYN SANGSTER, \\ KENNETH C.H. FEARON and JAMES A. ROSS
}

\begin{abstract}
Department of Clinical and Surgical Sciences (Surgery), University of Edinburgh, Royal Infirmary, Little France Crescent, Edinburgh, EH16 4SA, UK
\end{abstract}

Received January 8, 2009; Accepted February 11, 2009

DOI: $10.3892 /$ or_00000328

\begin{abstract}
The acute phase protein response (APPR) and peripheral blood mononuclear cell-derived inflammatory cytokine production was assessed in patients with advanced pancreatic cancer and age-matched healthy volunteers. We examined the relationship between the APPR, cytokine production and survival in these patients. Forty-two patients with pancreatic cancer cachexia and twelve age-matched healthy controls were recruited. The nutritional status, Karnofsky performance score, $\mathrm{C}$ reactive protein (CRP), serum interleukin-6, and in vitro monocyte interleukin-1 and interleukin- 6 production were measured. The dates of death of the pancreatic cancer patients were subsequently obtained and appropriate patient variables at baseline were entered into a Cox's proportional hazards model. The cancer patients had significantly lower: body mass index, Karnofsky performance score, serum albumin and elevated CRP and stimulated interleukin- 6 production. Both univariate and multivariate analysis demonstrated a strong association between tumour stage, CRP, stimulated interleukin-6 production and survival. Monocytes in cachectic pancreatic cancer patients are primed to produce high levels of interleukin-6 when stimulated. Overproduction of interleukin- 6 has a negative impact on survival. Decreased survival is associated with an elevated APPR. While the elevated APPR is probably related to locally produced interleukin- 6 in the liver, it seems possible that locally and systemically produced interleukin- 6 influences survival.
\end{abstract}

\section{Introduction}

There is increasing evidence that systemic inflammation (due in part to pro-inflammatory cytokines) may mediate a number

Correspondence to: Professor J.A. Ross, Tissue Injury \& Repair Group, Department of Clinical and Surgical Sciences (Surgery), University of Edinburgh, The Chancellor's Building, 47 Little France Crescent, Edinburgh, EH16 4SA, UK

E-mail: j.a.ross@ed.ac.uk

Key words: cachexia, cytokines, $\mathrm{C}$ reactive protein, interleukin-6 of the metabolic processes which contribute to cancer cachexia. We have demonstrated previously that a proportion of weight-losing pancreatic cancer patients have an elevated hepatic acute-phase protein response (APPR) (1) which has been shown to correlate with both increased resting energy expenditure and reduced duration of survival (2). Although a variety of factors, including cytokines and endocrine hormones, are known to influence hepatocyte protein metabolism, the principal regulator of the APPR in human hepatocytes is thought to be interleukin-6 (IL-6) (3). In our previous study (1) of patients with pancreatic cancer, however, no correlation was observed between serum concentrations of IL-6 and the presence of an APPR. Further investigation demonstrated a strong correlation between increased elaboration of IL- 6 by peripheral blood mononuclear cells (PBMC) of patients with pancreatic cancer and the presence of an elevated APPR (1), thereby suggesting that local rather than systemic, IL-6 levels might be important. The association between increased proinflammatory cytokine production, elevation of the acute phase response, hypermetabolism and shortened duration of survival in patients with pancreatic cancer therefore, makes the cytokine-hepatocyte axis a potential target for therapeutic intervention. This potential as a target in cachexia is increased further by the observation that pro-inflammatory cytokines may also contribute to the anorexia frequently observed in this syndrome (4-6).

In this study we examine the relationship between systemically and locally produced cytokines, the acute phase response, weight-loss and survival in a group of cachectic pancreatic cancer patients. Patients with advanced pancreatic cancer were studied as a model of cachexia as it is almost invariably present in this patient group (7). In addition, chemotherapy was not a standard treatment option during the study. Chemotherapy and its potential complications could not therefore act as confounding variables on weight loss, cytokine production, acute phase response or survival.

\section{Patients and methods}

Study design. The study was undertaken at one centre (Royal Infirmary of Edinburgh) on patients with advanced pancreatic cancer $(n=42)$. The control group were attending the hospital 
for elective surgical procedures. At baseline of the study, the weight, height, grip-strength and body composition of the patients were measured. Blood $(50 \mathrm{ml})$ was withdrawn. The blood was separated into serum, plasma and polymorphonuclear cell fraction as described below. Anthropometric measurements and blood samples were taken from each subject. The cancer patients were then followed up in the outpatient setting. The dates of death of patients were subsequently obtained from their primary care physician.

Cancer patients. Forty-two patients with unresectable pancreatic cancer who had been referred to the Royal Infirmary of Edinburgh were included in the study. Patients had lost $>5 \%$ of their pre-illness stable weight over the previous six months, had a Karnofsky performance score of 60 or more, and had a life expectancy greater than two months. All patients underwent staging by CT scan. Twenty-four patients had histological proof of diagnosis and eighteen patients were diagnosed on the basis of unequivocal clinical (operative) or radiological (CT scan) findings. Patients with pancreatic cancer were selected for this study as these patients usually experience severe weight loss associated with cancer cachexia. Patients were excluded if they had undergone surgery, endoscopic stenting, radiotherapy or chemotherapy during the previous four weeks, had other active medical conditions (e.g. major gastrointestinal disease, chronic renal failure, uncontrolled diabetes, and human immunodeficiency virus), a body mass index $(\mathrm{BMI})>30 \mathrm{~kg} / \mathrm{m}^{2}$ or had received medication which could profoundly modulate metabolism or weight, in particular, the use of fish oil or n-3 fatty acid preparations exceeding $200 \mathrm{mg} /$ day EPA or one capsule of fish oil/day within the previous 90 days. At the time of enrolment no patients had jaundice, pyrexia, severe anaemia, clinical or radiological evidence of infection and none were taking steroids at doses above that for physiological replacement. No patients had ascites or dependent ankle oedema. Pancreatic enzyme supplements were administered if patients had or developed clinical evidence of steatorrhoea. The Lothian Region Ethics Committee for human research approved the protocol, and written informed consent was obtained from all patients and controls. Procedures followed were in accordance with the Helsinki Declaration of 1975, as revised in 1983.

Healthy controls. Twelve age-matched healthy controls were recruited. These were patients admitted to the Royal Infirmary of Edinburgh for elective surgery for benign conditions: hernia repairs and gallbladder removal. All controls were weight stable. The same exclusion criteria were employed as those for the cachectic cancer patients.

Weight and body composition. At the initial assessment, preillness stable weight and duration of weight-loss were self reported. Patients height was measured and they were weighed on spring balance scales (Tanita Solar Powered Scale Model 1618, Tanita, Uxbridge, Middlesex, UK) without shoes and wearing light clothing.

Body composition was assessed using a Xitron Hydra multiple frequency bioelectrical impedance analyser (Xitron Technologies, San Diego, CA, USA) as previously described (8). Resistance was measured at 5 and $200 \mathrm{kHz}$. Total body water was derived using equations validated in a similar patient group (8). Lean body mass (LBM) was calculated assuming that lean tissue contains $73 \%$ water.

Physician-assessed physical function score. Patients were assessed for their level of physical function using the Karnofsky performance score.

Serum markers of systemic inflammation. Clotted blood $(10 \mathrm{ml})$ was centrifuged at 1,500 rpm for $30 \mathrm{~min}$ and separated serum stored as $1 \mathrm{ml}$ aliquots at $-70^{\circ} \mathrm{C}$ pending batch analysis at the study end. Serum concentrations of IL-6 (Quantikine, R\&D Systems, Abingdon, UK), and C reactive protein (CRP) (Dako, High Wycombe, UK) were measured by ELISA. Limits of detection were $0.5 \mathrm{pg} / \mathrm{ml}$ and $1 \mathrm{mg} / \mathrm{ml}$ respectively. Coefficient of variation for all assays was $<9 \%$ across the concentration range studied.

Isolation of PBMC. Peripheral blood nuclear cells (PBMC) were separated from $20 \mathrm{ml}$ of heparin anticoagulated blood on a hypaque gradient (Histopaque 1077, Sigma, Poole, UK) by centrifuging at $1,500 \mathrm{rpm}$ for $30 \mathrm{~min}$. Cells from the interface were removed and washed 3 times in cell culture medium [Rosswell Park Memorial Institute (RPMI) medium 1640, Life Technologies, Paisley, UK] with penicillin, streptomycin and glutamine (2 mmol/l) (Sigma) added. Cells were resuspended, counted and cultured in 96-well, flatbottomed tissue culture plates (Costar, Cambridge, MA, USA) at a concentration of $2 \times 10^{5}$ per well in $200 \mu \mathrm{l}$ cell culture medium with $10 \%$ fetal calf serum with penicillin, streptomycin and glutamine. PBMCs were then cultured in the presence or absence of $10 \mu \mathrm{g} / \mathrm{ml} \mathrm{E}$. coli lipopolysaccharide (Sigma, Poole, UK). Supernatants from PBMC cultures were removed at $24 \mathrm{~h}$ and stored at $-70^{\circ} \mathrm{C}$ for subsequent analysis.

Cytokine assays. IL-6 and IL-1ß concentrations from supernatants were measured by ELISA (Quantikine, R\&D Systems). Limits of detection were 240 and $195 \mathrm{pg} / \mathrm{ml}$ respectively for stimulated cells and 30 and $19.5 \mathrm{pg} / \mathrm{ml}$ respectively for unstimulated cells. Coefficient of variation for all assays was $<8.8 \%$ across the concentration range studied.

Plasma fatty acid analysis. Plasma was collected from the top of the hypaque gradients and stored as $5 \mathrm{ml}$ aliquots at $-20^{\circ} \mathrm{C}$ pending batch analysis at the study end. Analysis of EPA in patient plasma phospholipids before study commencement was performed by gas chromatography as described previously (9).

Statistical analysis. Results are expressed as mean (standard error of the mean: SEM). A Student's unpaired t-test for independent samples was used to look for differences between groups. Categorical variables on an ordinal scale (Karnofsky performance score) were analysed by the MannWhitney U test for unpaired samples. Correlations were examined using Pearson's method. Survival analysis was performed using Cox's proportional hazards model. For all tests, significance was considered at the $\mathrm{p}<0.05$ level. 
Table I. Baseline characteristics of 42 weight-losing patients with unresectable pancreatic cancer compared with 12 age matched healthy controls.

\begin{tabular}{|c|c|c|c|}
\hline & Cancer patients $(\mathrm{n}=42)$ & Healthy controls $(n=12)$ & $\mathrm{p}$-value \\
\hline $\operatorname{Sex}(M: F)$ & $22: 20$ & $5: 7$ & 0.532 \\
\hline Age (years) & $68(1)$ & $68(3)$ & 0.809 \\
\hline \multicolumn{4}{|l|}{ Stage of disease } \\
\hline II & 19 & NA & NA \\
\hline III & 10 & & \\
\hline IV & 13 & & \\
\hline Body mass index $\left(\mathrm{kg} \mathrm{m}^{-2}\right)$ & $21(1)$ & $27(1)$ & 0.001 \\
\hline Lean body mass (kg) & $43(1)$ & $47(2)$ & 0.197 \\
\hline$\%$ weight loss from usual weight & $21(1.1)$ & NA & NA \\
\hline \multicolumn{4}{|l|}{ Karnofsky performance score } \\
\hline 100 & - & 12 & 0.000 \\
\hline 90 & 5 & & \\
\hline 80 & 11 & & \\
\hline 70 & 18 & & \\
\hline 60 & 8 & & \\
\hline Plasma EPA (\%) & $0.88(0.09)$ & $0.71(0.16)$ & 0.361 \\
\hline $\mathrm{CRP}(\mathrm{mg} / \mathrm{l})$ & $22.9(7.0)$ & $1.7(0.2)$ & 0.004 \\
\hline Serum albumin $(\mathrm{g} / \mathrm{l})$ & $38(0.87)$ & $43(0.42)$ & 0.004 \\
\hline Serum IL-6 (pg/ml) & $18(6)$ & $5(2)$ & 0.057 \\
\hline Unstimulated PBMC IL-6 sups (pg/ml) & $3100(700)$ & $4100(900)$ & 0.410 \\
\hline Stimulated PBMC IL-6 sups (pg/ml) & $66200(6800)$ & $25200(3300)$ & 0.000 \\
\hline Unstimulated PBMC IL-1 sups (pg/ml) & $169(46)$ & $255(74)$ & 0.330 \\
\hline Stimulated PBMC IL-1 sups (pg/ml) & 3809 (454) & $3659(415)$ & 0.808 \\
\hline
\end{tabular}

Values are mean (SEM) or total number of patients; comparisons by Student's t-test for independent samples, or Mann-Whitney U-test for KPS; sups, supernatants.

\section{Results}

The characteristics of 42 weight-losing pancreatic cancer patients at baseline are shown in Table I. The cancer patients were elderly (mean age: $68 \mathrm{yrs}$ ) and were malnourished having lost, on average, $21 \%$ of their pre-illness stable weight and with a significantly reduced mean body mass index of 21. In general the patients' Karnofsky score was moderately impaired indicating that they were self-caring but unable to carry on normal activities or do active work. There was no difference in plasma EPA levels between cancer and healthy control patients.

The cachectic cancer patients exhibited an acute phase response with an elevated serum CRP and a trend towards elevated serum IL-6 levels (Table I). LPS-stimulated (but not unstimulated) PBMC from patients produced significantly higher levels of IL-6 compared with healthy controls. In contrast, both unstimulated and LPS-stimulated PBMC IL-1 production rates were not significantly different between the cancer patients and healthy controls.

CRP levels inversely correlated with Karnofsky performance score $(\mathrm{r}=-0.301, \mathrm{p}=0.027)$ and serum albumin $(\mathrm{r}=-0.419, \mathrm{p}=0.002)$. Serum CRP levels in the cancer patients and healthy controls $(n=54)$ correlated with PBMC IL-6 production rates in both unstimulated $(\mathrm{r}=0.582, \mathrm{p}=0.000)$ and stimulated $(\mathrm{r}=0.388, \mathrm{p}=0.005)$ conditions. There was no correlation between serum CRP levels and serum IL-6 $(\mathrm{r}=0.014, \mathrm{p}=0.920)$. There was no significant correlation between serum CRP and PBMC IL-1 production rates either in unstimulated $(\mathrm{r}=0.223, \mathrm{p}=0.063)$ or LPS-stimulated $(\mathrm{r}=0.223, \mathrm{p}=0.105)$ conditions.

Survival analysis was performed on the 42 cancer patients using Cox's proportional hazards model (Table II). Univariate analysis demonstrated a strong association between tumour stage, CRP and LPS-stimulated PBMC IL-6 production and survival. There was a trend between BMI and 
Table II. Survival analysis of 42 patients with unresectable pancreatic cancer using Cox's proportional hazards model.

\begin{tabular}{lcc}
\hline Variable & $\begin{array}{c}\text { Univariate } \\
\text { analysis }\end{array}$ & $\begin{array}{c}\text { Multivariate } \\
\text { analysis }\end{array}$ \\
\hline Sex & 0.415 & \\
Age & 0.664 & \\
Karnofsky performance status & 0.180 & \\
Stage & 0.005 & 0.004 \\
Body Mass Index & 0.059 & 0.032 \\
\% weight loss from usual weight & 0.267 & \\
Serum albumin & 0.562 & \\
CRP & 0.000 & 0.000 \\
Serum IL-6 & 0.226 & \\
Unstimulated PBMC IL-6 sups & 0.224 & \\
Stimulated PBMC IL-6 sups & 0.000 & 0.000 \\
Unstimulated PBMC IL-1 sups & 0.716 & \\
Stimulated PBMC IL-1 sup & 0.176 & \\
\hline
\end{tabular}

sups, supernatants

survival. Each of these factors retained a significant association with survival on multivariate analysis.

\section{Discussion}

Often used to describe severe weight loss, the word cachexia really represents a complex, multifactorial syndrome in which some or all of the following symptoms will be exhibited: anorexia, early satiety, hypophagia, fatigue, anaemia, oedema, and weight loss (characterised by excessive lean tissue wasting compared to undernutrition in an otherwise healthy individual). The average weight loss of the cancer patients was consistent with other studies of pancreatic cancer patients at diagnosis $(7,10)$. The significant impact cancer cachexia has on these patients resulted in a highly significant reduction in performance status from normal.

We have been interested in the potential anti-cachectic effects of the n-3 fatty acid eicosapentanoic acid (EPA) $(9,10)$. EPA and fish oils have been shown to reduce ex vivo stimulated cytokine production by peripheral blood mononuclear cells from healthy controls $(11,12)$ and patients with pancreatic cancer (13). EPA is present in small quantities in the average diet of our local population. Cancer cachexia may result in taste aversion to certain foods, anorexia and decreased overall calorie intake. We therefore were interested to compare plasma EPA levels in our cancer and control patients in case this might influence serum and in vitro PBMC produced cytokine levels. We found plasma EPA levels were the same between our healthy control and cancer patients.

Our results supported previous studies finding an association between cancer cachexia and an elevated acute phase response $(1,14,15)$. A variety of proinflammatory cytokines, including IL-6 and IL-1 have been implicated in the weightloss associated with cancer. The precise importance of their involvement remains unclear, but individually they will each induce anorexia, weight loss and an acute-phase protein response $(3,16,17)$. Circulating concentrations of proinflammatory cytokines are difficult to measure and interpret in human health and disease. In cancer cachexia, IL- 1 is infrequently detected, whereas IL-6 is more frequently found to be elevated $(18,19)$. Thus in the present study only serum IL-6 concentrations were measured. The trend towards elevated serum levels in the cancer patients would have been expected to reach significance had the cancer or control groups been larger. However, the lack of statistical significance in this sample size suggests that the association between elevated serum IL-6 level and advanced cancer may be relatively weak. It has been suggested that these proinflammatory cytokines act locally, and so measurement of ex vivo production may be a better guage of activity than circulating concentrations (1). In this study, ex vivo measurement demonstrated a highly significant elevation in supernatant IL-6 levels in LPS-stimulated PBMCs from cancer patients when compared to healthy controls. Conversely, PBMC supernatant levels of IL-1, while significantly elevated following LPS stimulation, did not differ between cancer patients and healthy controls. It may be that in pancreatic cancer cachexia PBMC production of IL-6 is upregulated while IL-1 production remains relatively normal. Alternatively PBMC IL-1 production could also be upregulated, but under these particular assay conditions the additional IL-1 remains in the PBMCs and is not released into the supernatant fluid.

An acute phase protein response is associated with, and may well be one of the most important, factors determining cancer cachexia (20). Fatigue and loss of muscle mass are hallmarks of cachexia. It was therefore unsurprising that CRP level correlated inversely with performance status. Serum albumin also correlated inversely with CRP levels. Lowserum albumin levels are frequently found in patients with acute inflammatory conditions including pancreatic cancer cachexia although the cause may be multifactorial (21). The principal regulator of the APPR in human hepatocytes is thought to be interleukin-6 (3). The strong correlation between CRP and PBMC-elaborated but not serum IL-6 suggests that local rather than systemic IL-6 may be responsible for hepatic acute phase protein production in cancer cachexia. Similarly, the lack of correlation between PBMC produced IL-1 and CRP suggests that PBMC produced IL-1 may not influence acute phase protein production in cachexia.

Cox's proportional hazards model (Table II) demonstrates advanced tumour stage to be an independent predictor of survival. Serum CRP level has previously been shown to be a highly significant predictor of survival in patients with pancreatic cancer cachexia, independent of tumour stage (2). Our results confirmed this. The production of acute phase proteins by the liver is associated with the mobilisation of peripheral amino acid stores principally from skeletal muscle. If this process is prolonged or excessive, it may lead to significant depletion of body energy and protein reserves and, thus, contribute to early death. 
As PBMC produced IL-6 correlated strongly with serum CRP levels, it is unsurprising that high PBMC produced IL-6 levels are also associated with decreased survival. There may be a direct association by IL- 6 contributing to the hepatic acute phase response. However, as both serum CRP and PBMC produced IL-6 remained independently associated with survival on multivariate analysis, locally produced IL-6 may have other effects in addition to stimulating the acute phase response which impact on survival.

It has previously been demonstrated in vivo that pancreatic cancers have increased expression of IL-6 mRNA (22). The same authors found that PBMC from cachectic pancreatic cancer patients were primed to produce a 14-fold higher level of IL- 6 mRNA when stimulated by IL- 6 producing pancreatic cancer cell lines compared to PBMC from noncachectic patients or healthy controls (22). This response was attenuated by anti-IL-6 antibodies. These authors suggested that pancreatic cancers were directly responsible for stimulating PBMC in cachectic patients. Our results suggest that IL- 6 produced by stimulated PBMC may directly influence survival in these patients. Further studies are required to elucidate the complex mechanisms underlying the acute phase response, cytokine production and survival in patients with pancreatic cancer cachexia.

\section{Acknowledgements}

We thank the patients for their commitment to the study. We also thank our clinical colleagues whose patients participated in the study (Professor O.J. Garden, Mr. K.K. Madhavan and Mr. Rowan Parks).

\section{References}

1. Falconer JS, Fearon KC, Plester CE, Ross JA and Carter DC: Cytokines, the acute-phase response, and resting energy expenditure in cachectic patients with pancreatic cancer. Ann Surg 219: 325-331, 1994.

2. Falconer JS, Fearon KCH, Ross JA, Elton R, Wigmore SJ Garden OJ and Carter DC: Acute-phase protein response and survival duration of patients with pancreatic cancer. Cancer 75: 2077-2082, 1995.

3. Castell JV, Gomez-Lechon MJ, David M, Fabra R, Trullenque R and Heinrich PC: Acute-phase response of human hepatocytes: regulation of acute phase protein synthesis by interleukin- 6 . Hepatology 12: 1179-1186, 1990.

4. Beutler B and Cerami A: Cachectin and tumour necrosis factor as two sides of the same biologic coin. Nature 320: 584-588, 1986.

5. Plata-Salaman CR: Central nervous system mechanisms contributing to the cachexia-anorexia syndrome. Nutrition 16 : 1009-1012, 2000 .

6. King PJ, Widdowson PS, Doods H and Williams G: Effect of cytokines on hypothalamic neropeptide $\mathrm{Y}$ release in vitro. Peptides 21: 143-146, 2000.

7. Wigmore SJ, Plester CE, Richardson RA and Fearon KC: Changes in nutritional status associated with unresectable pancreatic cancer. Br J Cancer 75: 106-109, 1997.
8. Hannan WJ, Cowen SJ, Plester CE, Fearon KC and deBeau A: Comparison of bio-impedance spectroscopy and multifrequency bio-impedance analysis for the assessment of extracellular and total body water in surgical patients. Clin Sci 89: 651-658, 1995 .

9. Wigmore SJ, Ross JA, Falconer JS, Plester CE, Tisdale MJ, Carter DC and Fearon KC: The effect of polyunsaturated fatty acids on the progress of cachexia in patients with pancreatic cancer. Nutrition 12: S27-S30, 1996.

10. Barber MD, Ross JA, Voss AC, Tisdale MJ and Fearon KC: The effect of an oral nutritional supplement enriched with fish oil on weight-loss in patients with pancreatic cancer. Br J Cancer 81: 80-86, 1999.

11. Endres S, Ghorbani R, Kelley VE, Georgilis K, Lonnemann G, Van der Meer JW, Cannon JG, Rogers TS, Klempner MS and Weber PC: The effect of dietry supplementation with n-3 polyunsaturated fatty acids on the synthesis of interleukin-1 and tumour necrosis factor by mononuclear cells. N Engl J Med 320: 265-271, 1989.

12. Meydani SN, Lichenstein AH, Cornwall S, Meydani M, Goldin BR, Rasmussen H, Dinarello CA and Schaefer EJ: Immunological effects of National Cholesterol Education Panel Step-2 diets with and without fish-derived n-3 fatty acid enrichment. J Clin Invest 92: 105-113, 1993.

13. Wigmore SJ, Fearon KCH, Maingay JP and Ross JA: Down regulation of the acute-phase response in patients with pancreatic cancer cachexia receiving oral eicosapentaenoic acid is mediated via suppression of interleukin-6. Clin Sci 92: 215-221, 1997.

14. Staal-van den Brekel AJ, Dentener MA, Schols AMWJ, Buurman WA and Wouters EF: Increased resting energy expenditure and weight loss are related to a systemic inflammatory response in lung cancer patients. J Clin Oncol 13: 2600-2605, 1995.

15. Wayman J, O'Hanlon D, Hayes N, Shaw I and Griffin SM: Fibrinogen levels correlate with stage of disease in patients with oesophageal cancer. Br J Surg 84: 185-188, 1997.

16. Moldawer LL, Andersson C, Gelin J and Lundholm KG: Regulation of food intake and hepatic protein synthesis by recombinant derived cytokines. Am J Physiol 254: G450-G456, 1988.

17. Hellerstein MK, Meydani SN, Meydani M, Wu K and Dinarello CA: Interleukin-1 induced anorexia in the rat. Influence of prostaglandins. J Clin Invest 84: 228-235, 1989.

18. Okada S, Okusaka T, Ishii H, Kyogoku A, Yoshimori M, Kajimura N, Yamaguchi K and Kakizoe T: Elevated serum interleukin-6 levels in patients with pancreatic cancer. Jpn J Clin Oncol 28: 12-15, 1998.

19. Martin F, Santolaria F, Batista N, Milena A, Gonzalez-Reimers E, Brito MJ and Oramas J: Cytokine levels (IL-6 and IFN-gamma), acute phase response and nutritional status as prognostic factors in lung cancer. Cytokine 11: 80-86, 1999.

20. Barber MD, Ross JA and Fearon KC: Changes in nutritional, functional, and inflammatory markers in advanced pancreatic cancer. Nutr Cancer 35: 106-110, 1999.

21. Fearon KCH, Falconer JS, Slater C, McMillan DC, Ross JA and Preston T: Albumin synthesis rates are not decreased in hypoalbuminemic cachectic cancer patients with an ongoing acutephase protein response. Ann Surg 227: 249-254, 1998

22. Martignoni ME, Kunze P, Hildebrandt W, Kunzli B, Berberat $P$ Giese T, Kloters O, Hammer J, Buchler MW, Giese NA and Friess H: Role of mononuclear cells and inflammatory cytokines in pancreatic cancer-related cachexia. Clin Cancer Res 11: 5802-5808, 2005. 